\title{
Tertiary Students' Preferences for Library Search Results Pages on a Mobile Device
}

\author{
Nicholas Vanderschantz ${ }^{1}$ [0000-0002-7729-7936], Claire Timpany ${ }^{10000-0003-0102-2191]}$, \\ and Chun Feng ${ }^{2}$ \\ 1,2 University of Waikato, Hamilton, New Zealand \\ ${ }^{1}$ \{vtwoz, ctimpany\}@waikato.ac.nz, ${ }^{2} \mathrm{cf} 37 @$ students.waikato.ac.nz
}

\begin{abstract}
Technology advancements and availability will result in library catalogues becoming more regularly accessed on small screen mobile devices in coming years with academics and students likely to be amongst the earliest adopters. There remain numerous unanswered questions regarding how to design library catalogues which offer effective library search on mobile devices. This broad research area requires the attention of $\mathrm{HCI}$, design, and reading researchers alike. This paper begins to address the user needs of library patrons when searching for books on a mobile device. Recommendations for mobile library catalogue design and further research is provided.
\end{abstract}

Keywords: Book Search, Digital Library Use, Library Catalogue Design, Mobile Interface Design.

\section{Introduction}

Tertiary students frequently search for printed books and eBooks both within the physical library as well as outside that library. These book searches are typically conducted using a digital library catalogue with a range of large screen and small screen technologies. The design of library catalogue interfaces will impact user success as well as user preference and therefore we suggest there is a need to investigate how tertiary students are searching for and using books during their educational pursuits.

This paper expands on the work reported in Vanderschantz et al., (2018) where we discussed the user habits and preferences of tertiary library patrons. Here we discuss our further investigation into the design of library search interfaces presented on mobile devices. A case study of present library catalogues and bookshop systems was used to inform the design of mobile interface prototypes. These prototypes were then used by students at two universities during an observed task study. Through this investigation we have identified mobile library catalogue features that impact user preferences.

\section{$2 \quad$ Related Work}

The work related to this investigation concerns the searching and browsing of digital libraries on mobile devices. The correlation of searching and browsing is discussed in 
the literature, for example Vemuri et al. (2006) identify that browsing and searching are fundamentally different activities, but argue that both fulfil important roles. Early work reported that browsing the surrounding shelves for books related to a user's search is a common serendipitous technique (Morse, 1971) used by information seekers in physical libraries (Bates, 1989). More recently Cooper \& Prager (2000) suggested that electronic retrieval of information may hinder these serendipitous opportunities and thus researchers (i.e. Su, 2005) suggest interface design metaphors such as the bookshelf metaphor may support serendipity in digital environments.

With increased prevalence of digital information seeking on small screen devices investigations of the use and usability of mobile digital library interfaces have begun. Liu et al. (2015) analysed mobile services provided by academic libraries of 100 universities. Wu et al. (2018) considered the use of library interfaces across multiple devices. Paterson et al. (2011) studied student attitudes towards mobile library services while our related study (Vanderschantz et al., 2018) reviewed interface preferences of students for libraries.

The remainder of this section briefly outlines the pertinent literature regarding choosing books and the display of search results.

\subsection{Choosing Books}

Hinze et al. (2012) note that when choosing books from physical and digital libraries, book-seekers typically follow a process that involves four main steps. We expand their description of these steps slightly and describe to include digital book selection, while their original description discussed physical books: (1) identifying books of interest via the catalogue or on the shelf, (2) retrieving those books physically or digitally, (3) selecting the books which best meet the book-seekers needs and finally (4) accessing the content within the book. These steps are similar to those summarised in a study by McKay et al. (2011) when looking at eBook selection in an academic library. They found that when people borrow or choose books from digital or physical libraries, their behaviour could be divided into three steps; gathering or collecting data to identify potential books, examining each book and evaluating relevance, then borrowing the books related to their information needs. Ross (2000) investigated choosing books for leisure reading rather than academic library search. That research suggests that when choosing books the process must include five relevant elements: reader's desired reading experience; alerts for new books available; the elements of a book that match the readers desired reading experience; the books clues to the reading experience that it offers; and the cost to the reader to get access to the book.

\subsection{Display of Search Results}

Shneiderman et al. (2000) suggest that central to the work of digital library researchers is the need to assist readers to find the resources they are looking for in a catalogue and arguably within a text. This requires investigation of how to design an overview of the information within the library or catalogue, how to create interfaces that users find intuitive, and usable, and how to effectively display search results for users. 
The search results page (SRP) is crucial for information seekers during book seeking and browsing. The SRP provides book information, lists of options, as well as bibliographic data, all of which will assist the user in making an informed decision. Mi \& Weng (2008) identified 10 important considerations for displaying the results of a book search that were often omitted from the interface or search result displays that they surveyed, including; item availability, book covers, intuitive navigation between results lists and individual records, and the ability to browse related results by call number.

There are few studies which have been conducted to understand preferences for the design of library interfaces. In one study, Merčun \& Žumer (2008) compared six library catalogues and found that none provided the same range of benefits included in the Amazon search interface. They concluded that the best interfaces provided features such as relevance ranking, faceted navigation, and supported browsing in their search results. Vanderschantz et al. (2015) also conducted a paper prototype study investigating design preferences for personal eBook libraries on mobile devices. The results of their study found that visual display of the book cover, book content, metadata, and book-related information all influenced reader preferences regarding the visual presentation of the interface.

\section{Case Study}

In 2017 an audit of the catalogue interfaces for six university libraries, two city libraries, three book retail websites, and one eBook catalogue was conducted to identify common interface features. A single keyword search for the term "design" was undertaken in each interface, from which an audit of the search results pages (SRPs) and the book result pages (BRPs) of each of the 12 interfaces was conducted. The interfaces were reviewed on both a desktop computer screen and a tablet device with negligible visual difference. We identified and classified the SRPs into four common design metaphors.

The two universities in China used the Text/Title metaphor, which listed search results in a numbered or ordered list with very little or no visual display of supporting information. Date, author, and publisher information was supplied by one of these two interfaces, while only date information was supplied in the second interface.

The three universities in NZ and the single university in the UK used interfaces with the Title/Cover metaphor in a list view. A small book cover was positioned with the book title in a numbered or ordered list format. All instances of the Title/Cover metaphor also comprised bibliographic information including authors, dates, and publisher information. When a cover was not available in the system a generic icon was used that depicted if the item was a book, article, or another form of resource in the catalogue.

One public library, and the Google Books interface used the Intro/Summary metaphor. This interface presented typical bibliographic information as well as summary or introductory information related to the book or article. Visual information in the form of a book cover was also present for both interfaces.

The Large Cover metaphor was identified for the three book retail websites audited. There was emphasis on the presentation of the cover of the book as a focal point resulting in pages that were presented as a list with fewer entries visible on a single screen 
without scrolling. To combat this, two of the three interfaces presented result entries in a grid pattern rather than a vertical list. All three of these interfaces included the book title and author as textual information alongside the large cover image. Only one of the Large Cover interfaces contained publisher information in the grid formatted list results and a different Large Cover interface included date information in a vertical list.

All of the interfaces audited used a vertical scroll and paged presentation of the results. The formatting for all of the library interfaces was a vertical list. Visual separation was created by negative space, background colours in a zebra striping, or a horizontal rule between entries. The bookstore catalogues were the only interfaces to present information in a grid view. It was not uncommon for cover information to be missing and for interfaces to contain no visual information in the SRPs. The amount of bibliographic information displayed by the different interfaces and within the identified categories was inconsistent and appeared to depend greatly on software developers rather than on user requirements or needs. All interfaces moved from an SRP to a single BRP when an item was selected from the search results list.

The findings of this case study were used to inform prototype design of test material for the research discussed in the following sections.

\section{$4 \quad$ Material Design}

To develop testing material for this study a typical design process was followed. Lawson (2005) explains that there is no single definition for what a design process is, but suggests that a design process involves the exploration of a range of potential solutions based on the problem that needs to be solved and the knowledge available. These potential solutions are refined to a point where they provide a solution to that problem. The process that was followed for this research was such that the design team first investigated and defined the problem, second developed initial design ideations, third developed and evolved the design ideations, and fourth iterated this process before settling on solutions to test. The findings of the visual audit of library and book retail interfaces along with findings derived from the related work were used to guide and develop the design of interface and interaction metaphors that could be tested as SRPs. Four central design metaphors were identified. A selection of the wireframe ideations for SRP designs are shown in Figure 1 and described in the following section.

\subsection{Initial Design Ideations}

Figure 1, SRP (1) is based on the very traditional vertical list format with a supporting book cover and metadata. We identified this design metaphor in the case study in both the Title/Cover and the Large Cover categories. The book cover metaphor is important to assist with searching as it provides a visual reference for recognition (Mi \& Weng, 2008) and was found by McKay et al. (2012) to influence length of use and loan times for eBooks. The information to be included in this type of SRP would be book title, author, publication date, and book location with either a small or large book cover as a supporting visual. 


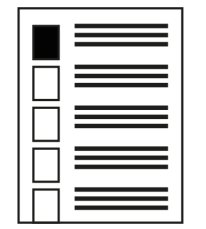

(1)

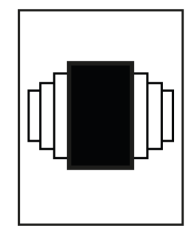

(3)

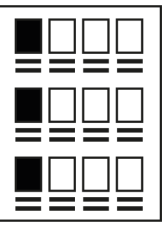

(2)

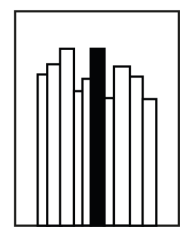

(4)

Fig. 1. Library SRP Interface Concepts (see Section 4.1)

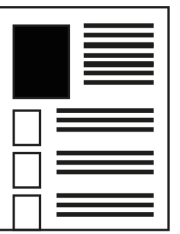

(5)

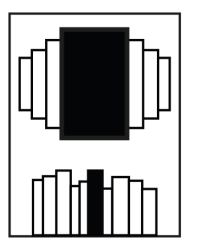

(7)

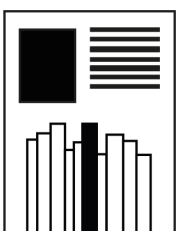

(6)

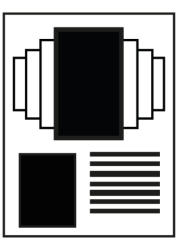

(8)
Fig. 2. Library SRP Interface Concepts (see section 4.2)

Figure 1, SRP (2) is based on the horizontal grid format with a supporting book cover and metadata. This SRP metaphor allows for less metadata and supporting information than SRP (1) due to the grid spacing that is available. In the case study we were able to identify that it was typical for title and author information to be presented, but not always in its entirety. A small or large cover graphic would support the textual information depending on the size of the grid cells implemented.

Figure 1, SRP (3) extends book cover use identified in the case study and incorporates the related work of Vanderschantz et al (2015) who investigated the use of a coverflow metaphor in library interfaces. This SRP does not easily allow for display of metadata that is not held on the cover of the book.

Figure 1, SRP (4) also extends the work of Vanderschantz et al (2015) who investigated the use of spines in digital library interfaces. This SRP does not easily allow for display of metadata that is not held on the spine of the book and contains a similar amount of information to that of the Title/Text interfaces identified in our case study.

\subsection{Developing \& Evolving the Design Ideations}

Here we describe the design work undertaken to develop and evolve the initial SRP interfaces illustrated in Figure 1. Traditionally the design of a library search result interface assumes a single transition from a search results page (SRP) to a book result page (BRP). That is to say, once a book or item is selected within a list, the search result page is replaced with a single book result page. This study is concerned with interfaces on a touch screen mobile device, therefore modern transitional screens typical of mobile device apps form a part of the design space for this problem. To investigate how transitional screens might extend the presentation of metadata, and visual or textual information, as well as provide opportunities for browsing of related search results these initial library SRP wireframes (Figure 1) were considered further. Figure 2 provides a 
selection of the enhancements that were identified for these original SRPs. The wireframe ideations in Figure 2 were hypothesized as being able to be used as SRPs that contained additional metadata and visual information. Further it was hypothesized that these screens could serve as transitional SRP's (tSRP) that would provide additional metadata on a single user tap of a book in the search result page list.

\subsection{Design Iteration}

Here we describe the design iteration that was undertaken after the initial investigation of SRP interfaces and tSRP interfaces in Figure 1 and Figure 2. While numerous SRP and tSRP were developed during the design ideation process only a small selection of these could appropriately be included in the initial user study (and detailed in Figures $1,2, \& 3)$. Through design iteration we resolved eight study prototypes for testing which can be divided into two equal groups. These prototypes included four apps that contained only an SRP, and a resulting BRP (labelled A1, A2, A3, A4). A second group of apps included an SRP, a resulting tSRP, and a resulting BRP. These prototypes were developed to extend prototypes A1-A4 and were labelled B1, B2, B3, B4.

Figure 3 shows design ideations for the SRPs (top) and corresponding tSRPs (bottom) used in the study reported here. For example, Figure 3 SRP (1) was used in our study for both prototype A1 and B1 with tSRP (a) being used in prototype B1. Equally, Figure 3 SRP (2) is used for both A2 and B2, with tSRP (b) being used in B2 etc.

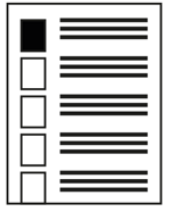

(1)

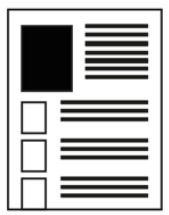

(a)

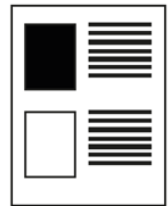

(2)

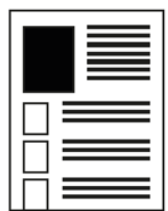

(b)

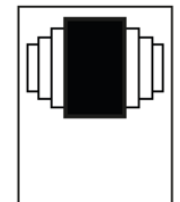

(3)

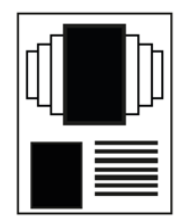

(c)

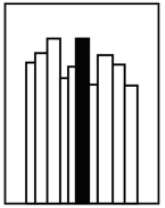

(4)

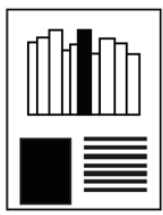

(d)

Fig. 3. Library SRP (top row) \& tSRP (bottom row) Interface Concepts

\subsection{Solutions to test}

Here we describe the design development that was undertaken to produce eight interactive interfaces that were able to be used in testing. The wireframe interfaces illustrated in Figure 3 were refined and implemented as design solutions with Figure 4 illustrating the design solution resolved on for prototype A1 and prototype B1. 
The prototypes that were developed for user testing incorporated realistic book covers, metadata, and related visual information. The prototypes were deployed as dynamic interactive mobile apps on an Android mobile tablet device.

Given the space we present in Figure 4 only the screens developed for prototypes A1 and B1. The green line in Figure 4 illustrates the user flow for prototype A1, while the blue line illustrates the user flow for prototype B1. On the left of Figure 4 is the search page used for every prototype in the study. When a search for the word "design" was fired using this left-hand screen (Figure 4), the user was presented with the top middle screen when they were using either prototype A1 or B1 (see Figure 3 SRP (1)). In prototype $\mathrm{A} 1$ if the user selected the top book, they would be presented with the farright screen, a BRP. In prototype B1, should the user select the top book on the SRP (top middle of Figure 4) they would be presented with the tSRP (bottom middle of Figure 4 and see Figure 3 tSRP (a)). Should the user select the top book again using the tSRP, they would be presented with the far-right BRP.

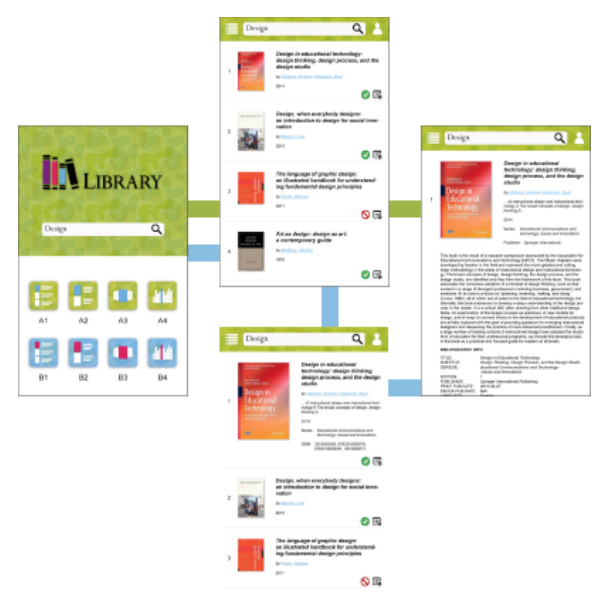

Fig. 4. Library SRP \& tSRP Interface Designs for prototype A1 and B1

\section{$5 \quad$ Method}

An observed task user study and guided interview were conducted with participants on two university campuses. The studies were conducted by a single researcher at one university in New Zealand and one university in China, typically inside or within the immediate vicinity of the university's library. Our researcher is fluent in both English and Chinese. Both libraries contained printed and digital documents in both English and Chinese, amongst other languages. Participants at the NZ university typically undertook their studies in English, while participants from the Chinese university might be taught in either English or Chinese.

The sample of 75 participants included 37 males and 38 females. 60 participants were surveyed at the NZ university and 15 at the university in China. A cross section 
of both undergraduate and postgraduate students at both universities agreed to participate. As per the institution's ethical approval, all participants received a verbal explanation of the study and completed a signed informed consent.

Participants were allowed as much time to explore and interact with the library search interfaces as each required. These interfaces were displayed on a Nexus7 ASUS tablet, a $200 \mathrm{~mm}$ tall by $114 \mathrm{~mm}$ wide by $8.65 \mathrm{~mm}$ thick device. No tuition was given for the use of these interfaces. Following the users' exploration of all eight of the prototypes the researcher conducted a guided interview to investigate the preferences and choices made by the participants. Field notes and interview recordings were made by the researcher by hand and were later manually coded and analysed.

\section{$6 \quad$ Results}

The results presented here from our observations and interviews give insight into the reading and search habits of our participants as well as their preferences for the visual designs that were tested. The results of the interview questions posed to participants regarding their current reading and library search behavior are discussed more fully in (Vanderschantz et al., 2018) - but will be referred to throughout this section to support the findings of the visual design preferences for search result pages.

\subsection{Perceived Importance of Interface Elements}

Importance of related books. 74 of 75 participants stated it was important when searching in a library interface that books relating to the topic of their current search be presented to them (Vanderschantz et al., 2018). Among these 74 participants, 57 thought related books would help them to find out what they want, 13 people thought it could give them more information for their search, and the remaining four participants believed related books provided further choices to assist with their search. The only participant, who thought related books were not important in the library search interface, explained "it gives too much information, and is not helpful for ... searching".

Importance of book descriptions. Participants were asked if the book description was important in the library search page. 71 of the 75 participants thought the book description was important. Among these 71 participants, 43 thought the book description could make choosing between books easier. 15 people thought that the description could clarify information they needed about the book to inform the decision to select or not select a specific book. 13 expected that the book description would provide additional related information during search to help identify topics and features of the books returned by the search.

Importance of book covers. Including the book cover on a library search page was considered to be important for $72 / 75$ participants (Vanderschantz et al., 2018). Among these 72 participants, 36 thought the book cover would make the book that they searched easy to find on the shelf. 15 assumed that the book cover could help them to decide if the book contains the information they are looking for. 11 participants thought 
it could give them more information, and the remaining ten participants believed the book cover improves the aesthetics of the interface, making it look "more interesting".

Importance of book spines. 43 participants considered the book spine important in the library search interfaces. 37 thought the book spine would help them to find the particular book that they would want to loan or read, and the other six participants thought it could give them clear information about the books available in the area of their search. From the 32 participants who thought the book spine was not important in the library search interface, 30 of them thought it was not likely to be helpful for the speed or accuracy of their searching, two of them described the book spines as hard to read, with P15 noting "too many choices, which does not help in searching for a book".

\subsection{Preference for the Design of Library Interfaces}

18 participants identified prototype B1 as their first choice and 16 participants chose A1. These two prototypes contained the same initial search results page, a traditional list view with a small supporting cover image for each list entry. B1 had a tertiary SRP with a larger cover image and some further metadata given when a search result was selected. A2 and B2 still rated relatively highly by participants with 13 and 7 participants respectively choosing these two prototypes as their preferred prototypes.

Taken together, the results for these four prototypes show the significance of the book cover when paired with metadata in a list view. This is reinforced by the qualitative feedback gained through the interviews. The most often given reason for choosing a preferred prototype was that the interface was considered easy for finding books (57\%). For example, when selecting NF A1 as their preferred prototype all 16 participants described this interface as easy. Eleven of the 18 participants who selected B1 and four of the 16 participants who selected A1 specifically detailed the information (metadata) given in the interface as "useful" and "helpful" to them while searching.

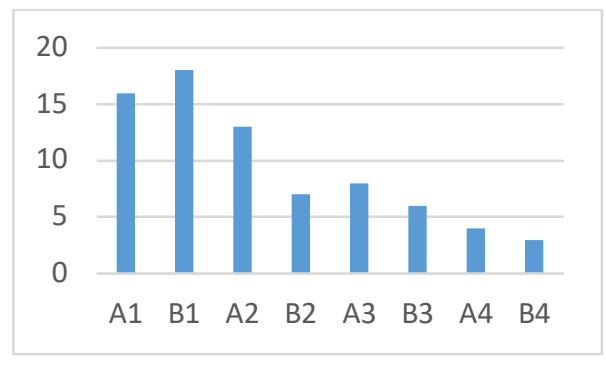

Fig. 5. Participant preference of library search prototype $(n=75)$

Perceived most useful prototype for searching. In the previous questions participants described their preference without any parameters, we next asked participants which prototype they found most useful for searching for a specific book. Similar to their preference for prototype, when asked specifically about the prototype that was most useful to them for searching, 18 participants considered B1, 14 participants A1, 11 participants A2 and six participants B2, as the most useful interface for their searching. 
$36 \%$ of the participants reported that their chosen interface gave clear information. Participant P9 explained that "I like the second page of B1, it shows me accurate information when I clicked on one book, and this is very helpful". While another participant P23 stated "the information in A1 is clear and enough" presumably suggesting that they are able to make a decision about a book without the additional metadata that is provided by the tertiary SRP provided by B1.

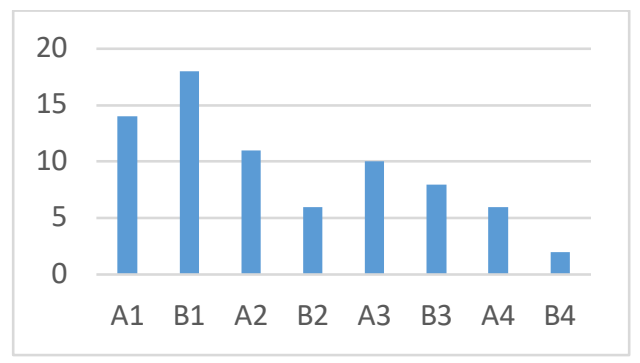

Fig. 6. Participant preference of library search prototype for searching for a specific book $(n=75)$

Perceived most useful prototype for browsing. Participants were asked which prototype they found most useful for browsing for a book. Differing from the answers given when asked about preference during search, when asked about browsing, greater numbers of participants preferred prototype A2. A2 provided users with less visible search results on a screen before scrolling was required yet provided more metadata per list entry than A1 provided. The second most often chosen prototype for browsing was B1 chosen by 18 participants. B1 provided more results on the initial search results page with limited metadata while the tertiary SRP for B1 provided additional metadata for the selected book while retaining further books in the list for consideration.

The most common reason given was that the interface provided clear information when browsing books (63\%). Sixteen of the 19 participants who selected A2 and 10/18 participants who selected B1 discussed the information provided as useful to them.

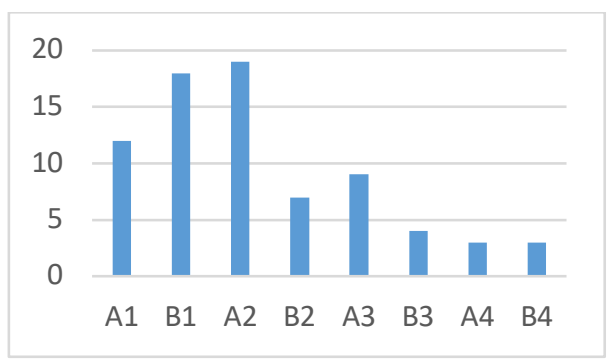

Fig. 7. Participant preference of library search prototype for browsing books $(n=75)$

Perceived most useful interface for providing information when browsing books. Participants were asked in which example they find the level of information most useful for browsing. 19 participants chose A1, 17 participants B1. 16 chose A2 and 8 chose 
B2. 54\% of the participants described their choice of prototype as providing clear information while $31 \%$ of the respondents believed the prototype they chose provided the right amount of information to be highly efficient for searching.

Perceived most useful for decision making. Participants were asked in which prototype they find the level of information most useful for making a decision. Participants tended to choose prototypes A1 and B1, 20 participants preferring B1 while 18 participants chose A1. Participants reported their interface of choice provided sufficient information for decision making or was efficient for browsing and searching.

Most intuitive transition from screen to screen during navigation. When a user tapped one of the four results entries in prototypes A1, A2, A3 or A4 they were presented with a book result page, however, when the user tapped a result in B1, B2, B3, or B4 they were presented with a tertiary search results page (as shown in Figure 4). 41 of the participants chose an interface that provided a tertiary search results page compared to 34 participants who chose an interface that did not provide a tertiary search results page before a book result page.

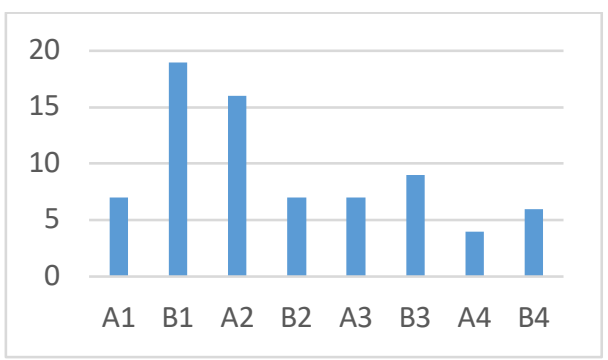

Fig. 8. Participant preference of library search prototype for intuitive navigation

\section{Discussion}

The 75 participants in this study browsed and borrowed from physical libraries regularly (Vanderschantz et al., 2018). The interactive prototypes that this study asked them to compare allowed us to assess the user preference for the display of search results and the importance of related books on user's preferences during their use of the systems. The somewhat traditional interface result list metaphor was preferred by participants in this study for all of the variables that we tested. Participants reported that these lists proved useful for searching or browsing and were intuitive and efficient interfaces to use. Participants often selected interfaces that provided additional information in either the SRP or the tSRP. This insight provides further evidence of the importance of the broader information provided by library search interfaces. Our implementation of tSRP provided opportunities to combine browsing and searching. One solution identified in the literature that looked at combining browsing and searching to meet information seeking needs was the ScentTrails project (Osten \& Lee, 2003). Our implementation of tSRP also provided the advantage of allowing the user to review a greater amount of metadata yet retain the ability to review the related books presented by the interface. This is likely akin to reviewing the back of a book or book metadata in the stacks of a 
physical library as identified by Hinze et al. (2012). The identified usefulness of tSRP by our participants supports the premise of Mi \& Weng (2008) that intuitive navigation between results lists and individual records is important.

The often-given reasons for participants preferences regarding the design of our prototypes were ease of book identification, clarity of information, assistance with book choice, and intuitiveness of the interaction and user experience. The metadata features most discussed as important by our participants were book cover, book title, author information, publication date, and blurb information. It has been reported widely that book covers effect user selection in physical libraries (Reutzel \& Gali 1998; Hinze et al. 2012; McKay et al. 2012; Stelmaszewska \& Blandford 2004) and bookshops (Buchanan \& McKay 2011). These preferences reinforce the findings of McKay et al. (2012) who noted that users read-time and tendency to abandon a book was influenced by inconsistencies present in the interface relating to metadata as well as cover presentation. Similar to our study, Vanderschantz et al. (2015) reported that users preferred an interface that displayed both a book cover and supporting bibliographic information.

The smaller numbers of participants at our Chinese university limits the ability to statistically analyse interactions that might be present between sites. Equally, this study did not allow for the testing of an interface that was in Chinese and how this might impact the results of the study. A broader case-study would be warranted to identify and examine instances of interfaces designed specifically for mobile screens and the metaphors and features that might benefit continued testing.

\section{Conclusion}

This paper explored interface and information presentation for library catalogues and digital libraries used by tertiary students. We executed an audit of existing library catalogue and bookshop interfaces to understand how best to develop the design of test material for our study reported here. Six university libraries, two city libraries, one eBook catalogue, and three book retail websites were reviewed. From the resulting set of design metaphors identified we created eight interactive digital prototypes for library search interfaces that were included in a user study with 75 participants at a university in NZ and a university in China. We conclude that across the range of identified design metaphors presently implemented by the library catalogues audited, the interfaces do not meet the needs or preferences of tertiary library catalogue users on mobile devices.

In analysing the interviews, we identified that most participants reported a preference for an interface that comprised book covers coupled with good quality bibliographic metadata. We recommend interfaces be designed to include the following information (in priority order); cover, title, author, publication date, publication information, library location information, as well as a book description. The interviews revealed that an interface that required the user to tap for additional information before proceeding to a traditional book result page was not seen as a hindrance to users. Therefore, if providing less information in an initial search result page will allow for presentation of a greater number of results on a screen, it is advised that a tertiary search result page be implemented with this additional metadata that is valued by the users. 


\section{References}

1. Bates, M. J.: The design of browsing and berrypicking techniques for the online search interface. Online review, 13(5):407-424. (1989).

2. Cooper, J.W. \& Prager, J.M.: Anti-serendipity: finding useless documents and similar documents, In Spragu, R.H. (Ed.), Proceedings of the 33rd Annual Hawaii International Conference on System Sciences, Maui, Hawaii, IEEE Computer Society, Piscataway, NJ. (2000).

3. Gaona-García, P. A., Martin-Moncunill, D., \& Montenegro-Marin, C.: Trends and challenges of visual search interfaces in digital libraries and repositories. The Electronic Library, 35(1):69-98. (2012).

4. Hinze, A., McKay, D., Vanderschantz, N., Timpany, C., \& Cunningham, S. J.: Book selection behavior in the physical library: Implications for ebook collections. In Proceedings of the 12th ACM/IEEE-CS joint conference on Digital Libraries, p.305-314. ACM, New York, NY. (2012).

5. Hinze, A., Vanderschantz, N., Timpany, C., Saravani, S-J., Cunningham, S. J., \& Wilkinson, C.: Use of Mobile Apps for Teaching and Research-Implications for Digital Literacy. In Proceedings of the International Conference on Asian Digital Libraries. p.173-184. Springer, Berlin, Heidelberg. (2017).

6. Lawson, B.: How designers think the design process demystified ( $4^{\text {th }}$ ed.). Elsevier/Architectural, Oxford; Burlington, MA. (2006).

7. Liu, Y. Q., \& Briggs, S. A library in the palm of your hand: mobile services in top 100 university libraries. Information technology and libraries, 34(2), 133-146. (2015).

8. Liu, Z.: Reading behavior in the digital environment: Changes in reading behavior over the past ten years, Journal of Documentation, 61(6):700-712. (2005).

9. Margam, M., \& Dar, S. A. Mobile Information Services and Initiatives in University Libraries: A New Way of Delivering Information. DESIDOC Journal of Library \& Information Technology, 37(2), 109. (2017).

10. McKay, D., Hinze, A., Heese, R., Vanderschantz, N., Timpany, C., \& Cunningham, S. J.: An exploration of ebook selection behavior in academic library collections. In International Conference on Theory and Practice of Digital Libraries p.13-24. Springer, Berlin, Heidelberg. (2012).

11. McKay, D., Buchanan, G., Vanderschantz, N., Timpany, C., Cunningham, S. J., \& Hinze, A.: Judging a book by its cover: interface elements that affect reader selection of ebooks. In Proceedings of the 24th Australian Computer-Human Interaction Conference. ACM, New York, NY, US. (2012).

12. Merčun, T. \& Žumer, M.: New generation of catalogues for the new generation of users: A comparison of six library catalogues, Program, 42(3):243-261. (2008).

13. Mi, J., \& Weng, C.: Revitalizing the library OPAC: Interface, searching, and display challenges. Information Technology and Libraries, 27(1):5-22. (2008).

14. Morse, P.M.: On browsing: the use of search theory in the search for information, Bulletin of the Operations Research Society of America, supplement, 19, p.1. (1971).

15. Olston, C. \& Chi, E. H.: ScentTrails: Integrating browsing and searching on the Web. $A C M$ Transactions on Computer-Human Interaction. 10(3):177-197. (2003).

16. Paterson, L., \& Low, B. Student attitudes towards mobile library services for smartphones. Library Hi Tech, 29(3), 412-423. (2011).

17. Ross, C. S.: Making choices: What readers say about choosing books to read for pleasure. The Acquisitions Librarian, 13(25):5-21. (2000). 
18. Shneiderman, B., Feldman, D., Rose, A., \& Grau, X. F.: Visualizing digital library search results with categorical and hierarchical axes. In Proceedings of the fifth ACM conference on Digital libraries (p.57-66). New York, NY: ACM. (2000).

19. $\mathrm{Su}, \mathrm{S}$.: Desirable search features of web-based scholarly e-book systems, The Electronic Library, 23(1):64-71. (2005).

20. Vanderschantz, N., Timpany, C., and Hinze, A.: Design exploration of ebook interfaces for personal digital libraries on tablet devices. In Proceedings of the 15th New Zealand Conference on Human-Computer Interaction, p.21-30. ACM. (2015).

21. Vanderschantz, N., Timpany, C., \& Feng, C. A Snapshot of Reading, Searching, and Browsing Preferences of Tertiary Students. In Proceedings of the 31st British Computer Society Human Computer Interaction Conference. BCS. (2018).

22. Vasileiou, M., Hartley, R. \& Rowley, J.: Choosing e-books: a perspective from academic libraries, Online Information Review, 36(1):21-39. (2012).

23. Vemuri, N. S., Torres, R. D. S., Fox, E. A., Fan, W., \& Shen, R.: Exploring digital libraries: integrating browsing, searching, and visualization. In Digital Libraries, 2006. JCDL'06. Proceedings of the 6th ACM/IEEE-CS Joint Conference on Digital Libraries. p.1-10. IEEE. (2006).

24. Wiederhold, G.: Digital libraries, value, and productivity. Communications of the ACM, 38(4): 85-96. (1995).

25. Wu, D., Liang, S., \& Bi, R. Characterizing queries in cross-device OPAC search: a largescale log study. Library Hi Tech, 36(3), 482-497. (2018). 